\title{
Inter-organ transmission of hepatocellular senescence induces multi-organ dysfunction through the TGF $\beta$ signalling pathway.
}

\section{Christos Kiourtis}

CRUK Beatson Institute https://orcid.org/0000-0002-6682-5668

Maria Terradas-Terradas

Cancer Research UK Beatson Institute

Lucy M. Gee

Newcastle University

Ya-Ching Hsieh

Cancer Research UK Beatson Institute

\section{Colin Nixon}

Beatson Institute for Cancer Research https://orcid.org/0000-0002-8085-2160

\section{William Clark}

Beatson Institute for Cancer Research

\section{Robin Shaw}

Beatson Institute

\section{Peter S. Hanson}

Newcastle University

\section{David Sumpton}

Cancer Research UK Beatson Institute https://orcid.org/0000-0002-9004-4079

\section{Gillian Mackay}

Beatson Institute for Cancer Research

\section{Stephanie May}

Cancer Research UK Beatson Institute https://orcid.org/0000-0003-0095-7403

\section{Miryam Müller}

Christian-Albrechts-University Kiel https://orcid.org/0000-0002-0751-1407

\section{Arafath K. Najumudeen}

Beatson Cancer Research UK Institute https://orcid.org/0000-0002-3764-5721

\section{Andrew Campbell}

CRUK Beatson Institute https://orcid.org/0000-0003-3930-1276

\section{Simon T. Barry}

Early Oncology, AstraZeneca,

\section{Christopher M. Morris}


Newcastle University

Fiona E. N. LeBeau

Newcastle University

Owen J. Sansom

Cancer Research UK Beatson Institute, Glasgow https://orcid.org/0000-0001-9540-3010

Kristina Kirschner

University of Glasgow https://orcid.org/0000-0001-7607-8670

Fiona Oakley

Newcastle University https://orcid.org/0000-0001-8692-1836

Thomas G. Bird ( T.bird@beatson.gla.ac.uk)

CRUK Beatson Institute

\section{Biological Sciences - Article}

Keywords:

Posted Date: December 14th, 2021

DOI: https://doi.org/10.21203/rs.3.rs-1133747/v1

License: (1) (1) This work is licensed under a Creative Commons Attribution 4.0 International License. Read Full License 


\section{Abstract}

Cellular senescence is associated with aging but also impacts various physiological and pathological processes such as embryonic development and wound healing. Factors secreted by senescent cells can affect their microenvironment, including local spreading of senescence. Acute severe liver disease is associated with hepatocyte senescence and frequently progresses to multi-organ failure. Why the latter occurs is poorly understood. Here, using genetic mouse models of hepatocyte-specific senescence, we demonstrate senescence development in extrahepatic organs and associated organ dysfunction in response to liver senescence. Additionally, we observe senescence-associated regeneration and reprogramming in the proximal tubules of the kidney. Using single cell transcriptomics and in vitro assays, we identify the Transforming Growth Factor $\beta$ (TGF $\beta$ ) pathway as a critical mediator of systemic spread of senescence. Lastly, TGF $\beta$ inhibition in our mouse models blocks senescence transmission to other organs and prevents renal dysfunction. Our results highlight the systemic consequences of organspecific senescence which, independent of aging, contributes to multi-organ dysfunction.

\section{Introduction}

Cellular senescence is a state of permanent cell cycle arrest accompanied by a hyper-secretory phenotype (Senescence-Associate Secretory Phenotype, or SASP), and is associated with both injury and agingrelated pathologies within affected organs ${ }^{1,2}$. Removal of senescent cells is beneficial to both organ function and organism survival ${ }^{3-6}$. Severe acute injury of any large organ is associated with systemic effects including multi-organ failure, of which acute liver failure (ALF) is a paradigm. ALF is itself associated with senescence induction and subsequent regenerative failure ${ }^{7}$. Studies both in vitro and in vivo have shown that senescence can be transmitted in a paracrine manner within affected organs ${ }^{8-11}$, however whether senescence can spread systemically to more distant organs remains unknown. Here we use acute liver senescence as an exemplar model, independent of systemic aging, to test whether senescence can be transmitted between organs in an endocrine manner. We demonstrate that systemic transmission of senescence affects multiple organs inducing target organ dysfunction, and identify the Transforming Growth Factor $\beta$ (TGF $\beta$ ) signalling pathway as a critical mediator of this process.

\section{Main Text}

\section{Liver senescence spreads to other organs and affects renal and brain function.}

To model tissue-restricted senescence we used a genetic mouse model of conditional, hepatocyte-specific excision of the p53-binding domain of MDM2 (Mdm2 ${ }^{\text {E5/E6fl; R26 }}$ LSL-tdTomato/LSL-tdTomato mice) (Fig. 1a). Intravenous administration of the hepatocyte-specific AAV8-TBG-Cre vector resulted in widespread genetic recombination in the hepatocytes $\left(\Delta \mathrm{Mdm} 2{ }^{\mathrm{Hep}}\right)$, unlike the empty vector AAV8-TBG-Null (control) (Fig. S1a, b). No significant Cre-mediated recombination (as manifested by the lack of red fluorescent 
protein (RFP) and p53 expression) occurred in extra-hepatic tissues, as previously reported ${ }^{12}$ (Fig. S1a, c, d). MDM2 inactivation resulted in p53 accumulation in the hepatocytes and subsequent upregulation of its target gene, the cell cycle inhibitor p21 which is itself a key marker of senescence (Fig 1b, c and Fig. $\mathrm{S} 1 \mathrm{a}, \mathrm{b})$. This in turn established a senescent phenotype in the liver manifested by increased SenescenceAssociated $\beta$-Galactosidase (SA $\beta-G a l)$ activity and a senescence-associated transcriptome (Fig. S2a-C).

We next examined other organs for senescence induction. In the kidney, we observed an increase in p21 expression and in SA $\beta$-Gal activity, along with a senescent transcriptional signature (Fig 1d, e and Fig. S2d, e). Additionally, kidneys of $\Delta \mathrm{Mdm} 2^{\mathrm{Hep}}$ mice contained increased concentrations of several SASP markers (Fig. S2f). In addition to the kidney, we observed increased expression of p21 in the brain and the lung of $\Delta \mathrm{Mdm} 2^{\mathrm{Hep}}$ mice (Fig. $1 \mathrm{f}-\mathrm{i}$ ). Therefore, in this model we observe renal, brain and lung senescence in response to genetically-induced acute hepatocellular senescence.

In $\Delta \mathrm{Mdm} 2^{\mathrm{Hep}}$ mice, p53 accumulation in hepatocytes also induces moderate liver injury and dysfunction as evidenced by an increase in plasma levels of Alanine aminotransferase (ALT), alkaline phosphatase (ALP) and bilirubin as well as an increase in hepatic cleaved caspase 3 (CC3, marker for apoptosis) (Fig. S3a-d). To address whether systemic transmission of senescence is driven by liver senescence or by liver injury, we induced liver senescence through oncogene activation. Using hepatocyte-specific expression of oncogenic KRAS ${ }^{612 D}$, we again observed liver senescence without histological or biochemical evidence of liver injury or dysfunction (Fig. $1 \mathrm{j}, \mathrm{k}$ and Fig. S3e- g). Similarly to the $\Delta \mathrm{Mdm} 2^{\mathrm{Hep}}$ model, renal p21 expression was increased in mice that expressed KRAS ${ }^{G 12 D}$ in their hepatocytes (Kras ${ }^{\mathrm{G} 12 \mathrm{D}}$ mice) compared to the control $\left(\mathrm{Kras}^{\mathrm{WT}}\right.$ ) mice (Fig. 1l, m). Therefore, we conclude that liver senescence, rather than liver injury, drives systemic spread of senescence.

\section{Liver senescence-induced organ senescence is associated with organ dysfunction.}

We then proceeded to explore whether renal and brain senescence were associated with dysfunction. To do this, we measured plasma Cystatin $\mathrm{C}$, a marker of renal filtration efficiency, and the levels of urine amino acids as a readout for renal tubular function. Plasma Cystatin $\mathrm{C}$ and several urinary amino acids were perturbed in the $\Delta \mathrm{Mdm} 2{ }^{\mathrm{Hep}}$ mice, consistent with renal dysfunction (Fig. $1 \mathrm{n}$, o and Fig. S4a). To assess brain functionality, we performed cognitive function studies. First, we performed the Y-maze test which assesses the natural instinct of mice for exploration. Normal murine behaviour consists of preferential exploration of the novel arm of a Y-maze after an acclimatisation period before opening the novel arm. $\Delta \mathrm{Mdm} 2{ }^{\mathrm{Hep}}$ mice spent significantly less time in the novel arm compared to control mice, consistent with impaired cognitive function (Fig. 1p and Fig. S4b). To further corroborate this finding, we performed electrophysiology on hippocampal slices from these mice and measured the gamma (30-80 $\mathrm{Hz}$ ) oscillation area power and frequency in response to a cholinergic agonist, carbachol. Following carbachol administration, brain sections from healthy mice produced network gamma oscillations of 
increasing power and stable frequency before stabilising after 120-150 minutes, as expected (Fig. S4c, d). In comparison, brain sections from $\Delta \mathrm{Mdm} 2^{\mathrm{Hep}}$ mice exhibited weaker and less stable oscillations consistent with abnormal hippocampal function (Fig. 1q and Fig. S4c, d). Hence, the senescence observed in the kidney and the brain in response to liver senescence is associated with organ-specific dysfunction.

\section{Liver senescence induces senescence-associated transcriptional changes and reprogramming at the proximal tubules.}

In order to uncover the changes occurring to the kidney in response to liver senescence, we performed single-cell RNA sequencing (scRNA-seq) on kidneys of $\Delta M d m 2^{\text {Hep }}$ and control mice (Fig. 2a). From a total of 30,236 single-cell transcriptomes and after stringent quality control, 24,215 cells were used for downstream analysis. Initial clustering of the control and $\Delta \mathrm{Mdm} 2^{\mathrm{Hep}}$ cells together resulted in 14 clusters (Fig. S5a), with each cluster containing cells both from $\Delta M d m 2$ Hep and from control mice. To obtain an accurate representation of the cell types of the normal kidney we clustered only the control cells initially. Using this method and by further sub-clustering of specific control clusters we were able to improve resolution, identifying some renal cell types which were not detected when clustering all cells from control and $\Delta \mathrm{Mdm} 2^{\mathrm{Hep}}$ together (Fig. S5b-d). Next, based on their transcriptome, the $\Delta \mathrm{Mdm} 2^{\mathrm{Hep}}$ cells were integrated in the UMAP of the control cells (Fig. S5e). Finally, cell types were assigned to each cluster and sub-cluster and the original UMAP was used for further analysis (Fig. S5f-h).

By colouring the cells on the UMAP depending on experimental cohort (control or $\Delta \mathrm{Mdm} 2^{\mathrm{Hep}}$ ), we observed that in the broad cluster of proximal tubular cells (PTC) particularly, the $\Delta M d m 2^{\text {Hep }}$ cells deviated from the control cells, consistent with a shift in their transcriptome (Fig. 2b). To further investigate this, we performed unsupervised pathway analysis in the PTC cluster, comparing $\triangle \mathrm{Mdm} 2^{\mathrm{Hep}}$ and control cells. This analysis revealed that several pathways associated with senescence (wound healing response and TGF $\beta$ production and downregulation of apoptosis) are enriched in the $\Delta \mathrm{Mdm} 2^{\mathrm{Hep}}$ PTCs (Fig. S6a). Next, to identify the cell identity of the p21-expressing cells we observed on kidney tissue, we created a p21 gene signature, comprised of genes associated with p21 expression. Using this signature we observed that most cells with a high score for this signature ( $\mathrm{p} 21^{\text {high }}$ cells) belong to the PTC compartment, in agreement with the p21 immunohistochemistry (IHC) on kidney sections which showed preferential localisation of the $\mathrm{p} 21^{+}$cells in the renal tubules (Fig. 2c). In addition, there was a significant increase of p2 $1^{\text {high }}$ cells in the $\Delta \mathrm{Mdm} 2^{\text {Hep }}$ PTCs (Fig. 2d), consistent with the increase in p21 ${ }^{+}$ kidney cells observed on tissue. An important function of PTCs is the uptake of filtered substances, including amino acids. As we observed suppression of some pathways related to polarity and amino acid transport in the $\Delta \mathrm{Mdm} 2^{\mathrm{Hep}}$ PTCs (Fig. S6a), we examined the expression of several transporters of the solute carrier (SIc) family across the different parts of the renal tubule. We observed marked differential 
expression of several Slc transporters specifically in the PTCs, but not in the distal tubular cells (DTCs) or in the Loop of Henle (LOH) (Fig. 2e). Some of these transporters (namely Slc6a6, Slc7a7, Slc25a39, Slc7a8 and Slc7a13) are involved in amino acid transport in the PTCs, consistent with the increase in amino acids observed in the urine of $\Delta M d m 2^{\mathrm{Hep}}$ mice ${ }^{13-15}$. We also observed that in the p21 high PTCs, the expression of several Slc transporter genes was altered compared to cells with negative or 0 score for the p21 signature (p21 low) PTCs (Fig. S6b). Therefore, the effects of liver senescence upon the kidney are particularly focused upon the PTCs, resulting in impaired PTC polarity and function.

Within the scRNA-seq dataset we identified an increase in proliferating PTCs in the Mdm2 ${ }^{\text {Hep }}$ kidneys (Fig. S7a, b). This was further validated by IHC for the S-phase marker BrdU which showed a significant increase of proliferating cells observed in the tubular cells of $\Delta \mathrm{Mdm} 2{ }^{\mathrm{Hep}}$ mice (Fig. S7c, d). Duplex immunofluorescence staining for $\mathrm{p} 21$ and BrdU confirmed that in $\Delta \mathrm{Mdm} 2{ }^{\mathrm{Hep}}$ kidneys the majority of the p $21^{+}$cells were not proliferating (Fig. S7e, f). Around $20 \%$ of p $21^{+}$cells incorporated BrdU, which could be explained by DNA repair taking place in those cells. Another interesting finding stemming from the scRNA-seq analysis was the upregulation of the reprogramming transcription factor SOX 9 by several $\triangle M d m 2$ Hep PTC, which was also validated by IHC (Fig. S7g-j). These observations are in agreement with studies demonstrating that SOX9 is a major driver of renal regeneration following injury ${ }^{16,17}$. In our case, however, only a small proportion (around 10\%) of proliferating cells expressed SOX9 (Fig. S7k, I). We observed a significant increase in expression of the kidney damage marker Lipocalin-2 (Fig. S8a, b) which was not, however, accompanied by evident manifestations of cell death as assessed by H\&E and CC3 IHC (Fig. S8c-e). Therefore, in addition to renal senescence, liver senescence induces reprogramming and regeneration to distinct subpopulations of the renal proximal tubular cells without evidence of cell death.

\section{SASP factors in the circulation of $\Delta M d m 2^{\text {Hep }}$ mice can induce senescence.}

To investigate the mechanism of senescence transmission from the liver to the kidney, we hypothesised that SASP factors secreted by the liver reach the kidney via the circulation. This hypothesis is supported

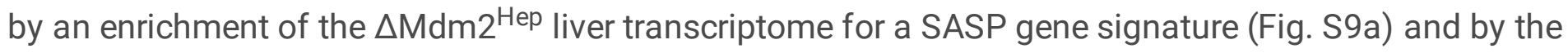
report that p21 is a major driver for the production of an early form of the SASP ${ }^{18}$. To test this hypothesis, we first treated cultured cells with plasma from $\Delta M d m 2^{\mathrm{Hep}}$ or control mice (Fig. 3a). We performed this using both wild type murine embryonic fibroblasts (wt MEFs) and neuronal cells differentiated from human pluripotent stem cells (iPSCs-derived neuronal cells). In both cases we observed a significant increase in SA $\beta$-Gal activity after treatment with $\triangle M d m 2^{\text {Hep }}$ plasma, but not with control plasma (Fig. $3 b$, c).

Next, we performed cytokine arrays on plasma from $\Delta \mathrm{Mdm} 2^{\mathrm{Hep}}$ and control mice. While plasma concentration of many of the screened factors remained unchanged (Fig. S9b), we observed a significant 
increase in the levels of 5 classical SASP markers: Transforming Growth Factor (TGF) $\beta 1$, TGF $\beta 2$, TGF $\beta 3$, C-C motif chemokine ligand 2 (CCL-2) and Leukaemia Inhibitory factor (LIF) in the plasma of $\triangle M d m 2^{H e p}$ (Fig. 3d). We referred to our renal transcriptomics analysis to assess cross-correlation to the downstream pathways (i.e. the TGF $\beta$ and the JAK-STAT signalling pathways) stimulated by these factors in the kidneys of $\triangle M d m 2^{\text {Hep }}$ mice. We first focused on the TGF $\beta$ pathway, since it was upregulated in the $\triangle \mathrm{Mdm} 2^{\mathrm{Hep}} \mathrm{PTCs}$ in our unsupervised GSEA on these cells (Fig. S6a). At the whole kidney level, the transcriptome of $\Delta M d m 2^{\text {Hep }}$ kidneys was enriched for a TGF $\beta$ signalling gene set (Fig. S9c) and this was further confirmed by western blotting for phosphorylated (p) Smad2 and pSmad3 (messengers of the canonical TGF $\beta$ pathway) on whole kidney lysates (Fig. 3e, S9d). At the single-cell level, we observed that specifically the $\triangle M d m 2^{\text {Hep }}$ PTCs had a higher score for a TGF $\beta$ gene signature compared to control PTCs and this was also validated on tissue with in situ hybridisation (ISH) for Smad7, a transcriptional target of TGF $\beta$ signalling (Fig. 3f, g). This corresponded with increased expression of the TGF $\beta$ receptor (TGF $\beta$ R1) in the kidney (Fig. S9e). The increase in plasma TGF $\beta$ ligands is associated with their increased hepatic production, as evidenced by their transcriptional upregulation in this organ (Fig. S2C and S9f).

Following a similar approach, we examined the LIF/JAK-STAT pathway, once again observing a transcriptional enrichment for a JAK-STAT pathway in $\triangle \mathrm{Mdm} 2^{\mathrm{Hep}}$ whole kidney lysates (Fig. S9g). This pathway was, however, principally upregulated in the mesenchymal cells and not in the PTCs (Fig. $3 \mathrm{~h}$, i). This was validated by IHC for PSTAT3 which confirmed the findings of the scRNA-seq data (Fig. 3h, j). Therefore, circulating SASP factors induced after hepatocellular senescence directly affect multiple renal populations including increased TGF $\beta$ signalling upon PTCs in $\triangle \mathrm{Mdm} 2^{\mathrm{Hep}}$ mice.

\section{Inhibition of the TGF $\beta$ pathway prevents systemic transmission of senescence.}

Having associated TGF $\beta$ pathway activation with senescence transmission, we went on to functionally test this pathway's role in senescence transmission, using an inhibitor of the TGF $\beta$ receptor 1 (TGF $\beta R 1 \mathrm{i}$, AZ12601011). We first tested this in vitro in plasma-treated iPSC-derived neuronal cells and observed a significant reduction in the number of SA $\beta-G a l^{+}$cells after TGF $\beta R 1 i$ treatment (Fig. 4a, b). Using the same inhibitor in $\triangle M d m 2^{\text {Hep }}$ mice (Fig. $4 \mathrm{c}$ ) resulted in inhibition of the TGF $\beta$ pathway in the kidney, as expected (Fig. 4d, S10a) and a significant reduction of $\mathrm{p} 21^{+}$cells in the kidney, brain and lung of these mice (Fig. 4e-h) without affecting the genetically induced liver senescence (Fig. S10b, c). A similar reduction in renal $\mathrm{p} 21^{+}$cells was also observed in the Kras ${ }^{\mathrm{G} 12 \mathrm{D}}$ model following TGF $\beta R 1 \mathrm{i}$ treatment (Fig. $\mathrm{S} 10 \mathrm{~d}, \mathrm{e})$. Furthermore, we observed a significant reduction in $\mathrm{BrdU}^{+}$and $\mathrm{SOX9^{+ }}$ renal tubular cells in the $\Delta \mathrm{Mdm} 2^{\mathrm{Hep}}$ mice, suggesting an overall amelioration of the reprogramming and regeneration phenotype observed in the $\Delta \mathrm{Mdm} 2^{\mathrm{Hep}}$ kidneys, despite the unaltered levels of renal Lipocalin-2 (Fig. S10fi). The improvement in senescence and associated reprogramming/regeneration phenotype was 
accompanied by a significant improvement in renal function, as indicated by a significant reduction of plasma Cystatin $\mathrm{C}$ and urine amino acids (Fig. 4i, j and Fig. S10j). We therefore conclude that inhibition of the TGF $\beta$ signalling pathway partly prevents the systemic transmission of senescence and the associated reprogramming and regeneration phenotype in the kidney.

\section{Discussion}

The SASP is a central mediator of the non-autonomous effects of senescent cells. Here we present evidence that senescence can be transmitted to and affect the function of distant organs in a systemic manner. In the context of acute injury, senescence has often been described as part of a finely-tuned mechanism with overall beneficial effects for wound healing ${ }^{19,20}$. As described by others ${ }^{21-23}$, SASP factors are able to induce reprogramming in neighbouring cells, facilitating tissue regeneration. However, following severe injury, this mechanism may have the opposite effect, through excessive SASP production, including senescence- and reprogramming-inducing factors. This excess of SASP factors may enter the circulation and induce widespread senescence and reprogramming to distant organs. In turn, this excessive stimulus for senescence, re-programming and regeneration can compromise organ function.

Systemic transmission of senescence may be relevant to several diseases. Here we use a model of hepatocyte-specific senescence to model an acute senescence phenotype, such as the one observed during ALF. ALF is itself characterised by sequential multi-organ failure typically beginning with the kidney and also involving the brain and lung in addition to other organs. This clinical progression may, at least in part, be underpinned by the systemic transmission of senescence. The observation that TGF $\beta$ signalling is a central driver of systemic transmission of senescence paves the way for new therapeutic approaches in diseases where this phenomenon occurs. This is in line with the beneficial effects of senolytics and senomorphics that have been elegantly demonstrated on numerous pathologies ${ }^{5,24-26}$. In conclusion, our results demonstrate that systemic transmission of senescence can induce systemic organ dysfunction which may be central to mutlisystemic sequelae in many diseases.

\section{Declarations}

Author contributions

C.K. contributed to the conceptualisation of the project, designed and performed animal studies, designed and performed experiments, analysed data, created the figures and wrote the manuscript (original draft and subsequent editing). M.T.T. performed the bioinformatics analysis of the single-cell RNA sequencing (scRNA-seq) data and created figures. L.G. performed animal studies and experiments and analysed data. YC.H. and K.K. performed the scRNA-seq. C.N. performed immunohistochemistry stainings. W.C. performed the bulk RNA sequencing (RNA-seq). R.S. performed the analysis of the bulk RNA-seq. D.S. and G.M. performed the metabolomics experiments. S.M. and M.M. assisted with animal experiments. A.K.N. assisted with experiments. F.E.N.L. and F.O. designed experiments and analysed data. A.C., O.J.S. and 
S.T.B. provided resources. T.G.B. contributed to the conceptualisation of the project, assisted with data analysis and figure generation, edited the original draft, provided resources and acquired funding. All authors contributed to the drafting and editing of the manuscript.

Acknowledgements

We would like to thank the CRUK Beatson Institute's histological services, biological services and molecular technology, metabolomics and bioinformatics services, central services as well as the Veterinary Clinical Pathology Lab (University of Glasgow) for their help.

Funding

C.K., YC.H., W.C., C.N., R.S., D.S., G.M., S.M. and A.C. were funded by Cancer Research UK (Grant number: A17196). K.K. was funded by a John Goldman Fellowship sponsored by Leukaemia U.K.

(2019/JGF/003). M.T.T. was supported by MRC funded Ph.D. studentships (MR/N013166/1). L.M.G was funded by a Ph.D. studentship from the National Institute for Health Research (NIHR) Newcastle Biomedical Research Centre (BRC). A.K.N was supported by the CRUK Rosetta Grand Challenge grant (A25045). F.O. was funded by the MRC (grants MR/K0019494/1 and MR/R023026/1). M.M. and T.G.B. were funded by the Wellcome Trust (Grant number: WT107492Z) and CRUK HUNTER Accelerator Award (Grant number: 175 A26813). O.J.S. was funded by Cancer Research UK (Grant number: A21139).

Competing interest statement

S.T.B. is an employee and shareholder of AstraZeneca.

Additional information

Supplementary Information is available for this paper.

Correspondence and requests for materials should be addressed to $\mathrm{Dr}$ Tom G Bird.

Data availability

The fastq files and processed data for the single-cell RNAseq analysis of mouse kidney cells can be found on the Gene Expression Omnibus (GEO) repository; accession number: GSE189726. All other data generated and/or analysed during the current study are available from the corresponding author on reasonable request.

Code availability

No custom code or mathematical algorithms were used in this manuscript

\section{Materials And Methods}




\section{Animal studies}

All mouse experiments were carried out in accordance with the UK Home Office regulations (licence 70/8891; protocol 2 or licence PP0604995; protocol 3) and mice were housed in a licenced, pathogen-free facility, under a $12 \mathrm{hr}$ light-dark cycle, at stable temperature $\left(19-23^{\circ} \mathrm{C}\right)$ and humidity $(55 \pm 10 \%)$. The mice were bred on a mixed C57BI6 background, were housed in conventional cages and had ad libitum access to food and water. All experiments were performed on 8-12 weeks old male and female mice according to the guidelines of the Animal Welfare and Ethical Review Body (AWERB) and were in agreement with the ARRIVE guidelines ${ }^{27}$.

Treatment with AAV was performed as described previously ${ }^{12}$. Briefly, mice were injected with $2 \times 10^{11}$ genomic copies (GC) of either (AAV8.TBG.PI.Cre.rBG (AAV-Cre) (Addgene, 107787-AAV8) or AAV8.TBG.PI.Null.bGH (AAV-Null) (Addgene, 105536-AAV8) in 100 $\mu$ sterile PBS via a single tail vein injection. Mice in this study weighed $21.9-31.9 \mathrm{~g}$ at the time of induction.

For the mouse model of hepatocyte-specific inactivation of Mdm2, male mice homozygous for the Mdm2tm2.1Glo allele (ID: MGI:2385439176 ${ }^{28}$ ) and the Gt(ROSA)26Sortm14(CAG-tdTomato)Hze allele

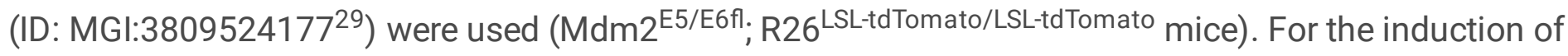
Ras-induced liver senescence, 8-12 weeks old male and female mice heterozygous for the Kras ${ }^{\text {tm }} 4 \mathrm{Ty}$ allele, (ID: MGI:242994817830) and the Gt(ROSA)26Sortm14(CAG-tdTomato)Hze allele (ID:

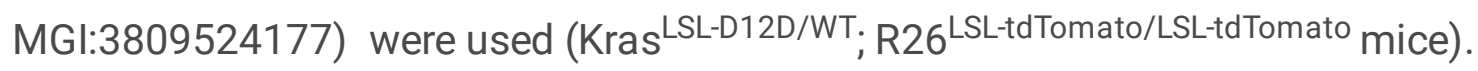

For treatment with TGF $\beta R 1 \mathrm{i}$, the mice received either TGF $\beta R 1 \mathrm{i}$ or Vehicle $(0.5 \% \mathrm{HPMC} / 0.1 \%$ Tween 80$)$ by oral gavage twice daily. The dose of TGFßR1i was $50 \mathrm{mg} / \mathrm{kg}$ in $100 \mathrm{ml} \mathrm{PBS}$.

Mice were euthanised by $\mathrm{CO}_{2}$ inhalation in a $\mathrm{CO}_{2}$ chamber, cervically dislocated and then weighed. Blood was collected immediately via cardiac puncture for whole blood analysis (EDTA-coated tubes; Sarstedt) and plasma biochemistry (Lithium-heparin coated tubes; Sarstedt). Plasma was separated by centrifugation ( $2350 \mathrm{~g}$ for 10 minutes at room temperature) within 1-3 hours after culling and stored immediately at $-80^{\circ} \mathrm{C}$. After weighing the liver, the caudate and left median lobes lobe were snap-frozen on dry ice for protein/RNA extraction and for histology studies respectively. The remaining liver was fixed in $10 \%$ neutral buffered formalin (NBF) for $22-24$ hours before transfer to $70 \%$ ethanol for further processing. The left kidney was immediately cut in half and both halves were snap frozen on dry ice for protein/RNA extraction and for histology. The right kidney, heart, brain and lungs were fixed in $10 \% \mathrm{NBF}$ for $22-24$ hours and then changed to $70 \%$ ethanol.

\section{Assessment of cognitive function}

\section{Y-maze test}


The mice were individually placed into a testing area measuring $25.66 \times 17.53 \mathrm{~cm}$ onto a Samsung Galaxy Tab 210.1 for 5 minutes. Steps and distance travelled were recorded using MouseTrapp software. The $Y$ maze arena had three arms of $40 \mathrm{~cm}$ identified as $A 1 \mathrm{~A} 2 \mathrm{~A} 3$, each with a differentiating marker at the end of the arm. Mice were assigned different start arms in a rotating allocation, and tested prior to the start of the experiment and on day 4, with differing arm allocations each time. In the T1 phase mice were placed into the maze with only two arms open for 5 minutes to explore the arena. The mice were then removed to a clean cage for 1 minute (fresh cage used per cage of mice). Then in the T2 phase mice were returned to the maze in the starting position with the novel arm opened for 2 minutes, and allowed to explore. A camera was set up above the maze to record movements and the video files analysed via Ethovision XT13 software.

\section{Brain slice electrophysiology}

Animals were killed humanely by anaesthetic overdose with inhaled isoflurane and intramuscular injection ketamine ( $\geq 100 \mathrm{mg} / \mathrm{kg})$ and xylazine $(\geq 10 \mathrm{mg} / \mathrm{kg})$ as previously described ${ }^{31}$. The mice were then transcardially perfused with at least $25 \mathrm{ml}$ of sucrose rich artificial cerebral-spinal fluid (SaCSF) composed of (mM) 252 sucrose, $3.0 \mathrm{KCl}, 1.25 \mathrm{NaH}_{2} \mathrm{PO}_{4}, 24 \mathrm{NaHCO}_{3}, 2.0 \mathrm{MgSO}_{4}, 2.0 \mathrm{CaCl}_{2}$ and 10 glucose. The brain was removed and sliced at $450 \mathrm{~mm}$ horizontal slices with a Leica VT1000S vibratome in ice cold SaCSF. Slices were trimmed to the hippocampal region and maintained at $32-34{ }^{\circ} \mathrm{C}$ at an air liquid interface between normal aCSF (sucrose replaced with $126 \mathrm{mM} \mathrm{NaCl}$ ) and humidified 95\% $\mathrm{O}_{2} / 5 \%$ $\mathrm{CO}_{2}$. Oscillations were evoked with $10 \mu \mathrm{M}$ cholinergic agonist carbachol, to activate transmission through acetylcholine receptors. Extracellular recording electrodes were filled with normal aCSF (resistance 2-5 $M \Omega$ ), and field recordings taken from the border between stratum radiatum and stratum lacunosum moleculare in CA3. Recordings were taken with Axoclamp-2B amplifier (Axon Instruments Inc., Union City, CA, USA) and extracellular data filtered at 0.001-0.4 kHz low-using Bessel filters. Mains noise was deducted with a Humbug (Digitimer, Welwyn Garden City, Herts, UK), and data redigitized at $10 \mathrm{kHz}$ using an ITC-16 interface (Digitimer, Welwyn Garden City, UK). Axograph 4.6 software (Axon Instruments Inc., Union City, CA, USA) was used for data acquisition and analysis.

To generate power spectra Axograph used Fourier analysis using 60 seconds per 10 minute recording. This was used to calculate peak frequency and area power (area under the peak). Mouse gamma frequency oscillation was measured at frequencies between 15 and $49 \mathrm{~Hz}$. Oscillations were categorized as stable when area power measured within $10 \%$ for three consecutive 10 min recording intervals.

\section{Histology}

FFPE sections, $4 \mu \mathrm{m}$ thick, were used for simple immunohistochemistry $(\mathrm{IHC})$ and for multiplex immunofluorescence (IF). Sections were subjected to heat-induced antigen retrieval, followed by protein 
blocking to reduce non-specific staining. Incubation with primary antibody overnight at $4{ }^{\circ} \mathrm{C}$ or for 1 hour at room temperature was followed by secondary antibody incubation and signal detection. Details on the antibodies can be found in Supplemental Table 1.

Photos were taken with a Zeiss Axiovert 200 microscope using a Zeiss Axiocam MRc camera. Stained slides were scanned using a Leica Aperio AT2 slide scanner (Leica Microsystems, UK) at 20x magnification. Automated quantification of positively stained cells or area was performed using the HALO image analysis software (V3.1.1076.363, Indica Labs). Manual quantification of $\mathrm{p}^{2} 1^{+}$and BrdU ${ }^{+}$ kidney cells was performed on 20 random fields at 20x magnification. For p21 IHC quantification on brain sections, total brain area was calculated using the HALO software and the $\mathrm{p} 21^{+}$cells were manually counted in the whole brain tissue area.

For multiplex IF, $4 \mu \mathrm{m}$ tissue sections underwent heat-induced antigen retrieval (HIER) by boiling (in a waterbath) in citrate buffer (10mM Na Citrate (Sigma, W302600), 0.05\% Tween 20 (Sigma, P1379), pH 6) for 25 minutes, and were subsequently cooled down for 20 minutes in the retrieval solution. Peroxidase quenching with $3 \% \mathrm{H}_{2} \mathrm{O}_{2}$ (Sigma, 95321) was followed by biotin (Invitrogen, 4303) and protein blocking (Abcam, ab64226). Sections were incubated with the primary antibodies either for 1 hour at room temperature, or overnight at $4{ }^{\circ} \mathrm{C}$, followed by 45 minutes with the secondary antibodies (conjugated to fluorophor) together with DAPI $(1 \mathrm{mg} / \mathrm{ml})$. Sudan black B was then used to quench autofluorescence. An aqueous mounting solution (DAKO, S1964 was used for mounting.

The anti-p21 antibody required additional signal amplification which was achieved by using the tyramide signal amplification (TSA) system. Briefly, after incubation with the anti-p21 primary antibody, the sections were incubated with a secondary anti-rat biotinylated antibody for 30 minutes, followed by 30 minute incubation with an avidin-HRP complex (Vectastain ABC, Vector, PK-7100). Then, the sections were incubated with TSA FITC (Perkin Elmer, NEL741B001KT) for 6 minutes (in the dark). After that, the sections were subjected to a 5 minute HIER in order to remove the anti-p21 antibody complex, underwent protein blocking and then were incubated with the other primary antibodies as described in the previous paragraph. Images were taken using a Zeiss 710 upright confocal Z6008 microscope. The Opera Phenix scanner (Perkin Elmer) was used to scan the stained sections at 20x magnification. For the analysis of scanned sections, the Columbus software (Perkin Elmer) was used to create a custom algorithm to quantify 20 random fields of view.

In situ hybridization (ISH) was performed in an autostainer (Leica Bond $\mathrm{R}_{\mathrm{x}}$ ) using the 2.5 LSx RNAScope kit (Bio-Techne, 322700) according to the manufacturer's instructions. Probes against Smad7 mRNA (BioTechne, 429418), TGF $\beta 1$ (Bio-Techne, 407758), TGFß2 (Bio-Techne, 406188) and TGF $\beta R 1$ (Bio-Techne, 431048) were used for the detection of the respective mRNA, and PPIB (Bio-Techne, 313918) as a positive control of gene expression.

\section{Senescence-associated $\beta$-Galactosidase staining}

Page $12 / 28$ 
Staining for senescence-associated $\beta$-Galactosidase (SA $\beta$-Gal) was performed as described previously 32 . Briefly, $10 \mathrm{~mm}$ thick cryosections or cultured cells were fixed in $2 \%$ paraformaldehyde/ $0.25 \%$ glutaraldehyde in PBS for 15 or 5 minutes respectively. This was followed by three washes with PBS $1 \mathrm{mM} \mathrm{MgCl} 2$ ( $\mathrm{pH} 5.5$ or 6 for mouse or human cells/tissues respectively) and incubation with staining solution $\left(1 \mathrm{mM} \mathrm{MgCl}_{2}, 0.5 \mathrm{mM} \mathrm{K}_{3} \mathrm{Fe}(\mathrm{CN})_{6} 0.5 \mathrm{mM} \mathrm{C}_{6} \mathrm{FeK}_{4} \mathrm{~N}_{6} \star 3 \mathrm{H}_{2} \mathrm{O}\right.$ and $1 \mathrm{mg} / \mathrm{ml} \mathrm{X-Gal} \mathrm{in} \mathrm{PBS,} \mathrm{pH} 5.5$ or 6 for mouse or human cells/tissues respectively) overnight (liver sections, cells) or for 2.5 hours (kidney sections). After three washes with PBS, cryosections were counterstained with eosin and mounted, while cells (on coverslips) were mounted immediately. For quantification, SA $\beta-G a l^{+}$and SA $\beta-G a l^{-}$cultured cells were counted in 20 random fields of view.

\section{RNA extraction}

Whole tissue RNA was extracted using the Qiagen RNeasy kit (Qiagen, 74104), including the optional DNase step, as described previously ${ }^{12}$. Briefly, 20-30mg of snap frozen tissue were homogenized in $600 \mathrm{ml}$ buffer RLT/1\% $\beta$-mercaptoethanol using the Precellys Evolution homogenizer (Bertin

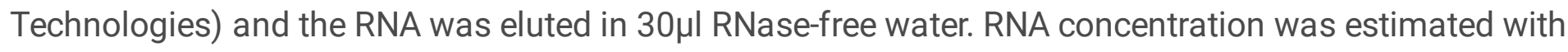
the Nanodrop 2000 and only samples with a $260 / 280$ ratio $\geq 2$ were used for further analysis.

\section{cDNA generation and qPCR}

cDNA was generated using the QuantiTect Reverse Transcription Kit (Qiagen, 205311) according to the manufacturer's instructions from $1 \mu \mathrm{g}$ RNA. A PTC-200 Gradient cycler (MJ Research) was used to perform the gDNA wipeout and reverse transcription steps. A sample-free reaction and a reaction without the reverse transcriptase served as negative controls. Quantitative real time PCR was performed with the SYBR Green system (Qiagen, 204145)using a QuantStudio 5 Real time PCR system in a 384 well plate setting (final reaction volume $10 \mu \mathrm{l}$ per well). All primers used were purchased from Qiagen, as shown in Supplemental Table 2. Each biological replicate (mouse) was run in triplicate and the $18 \mathrm{~S}$ ribosomal RNA (Rn18S) was used as a house keeping gene for normalization. Relative expression was calculated using the $\Delta \Delta$ Ct method.

\section{Whole tissue (bulk) RNA sequencing}

For bulk RNA-sequencing (RNA-seq), RNA was extracted as described above. Briefly, the RNA was tested on an Agilent 2200 TapeStation (D1000 screentape) using RNA screentape and only samples with a RIN $>7$ were further processed for library preparation. 20ng/ml of RNA were used to prepare libraries using the TruSeq Stranded mRNA Kit. Agilent 2200 Tapestation was used to assess library quality and Qubit (Thermo Fisher Scientific) was used to check concentration. The libraries were then run on the lllumina NextSeq 500 using the High Output 75 cycles kit (paired end, 2x36 bp cycle, dual index ( 15 and 17 Illumina)). 
For the bioinformatics analysis, raw data quality checks and trimming were performed using FastQC version 0.11.7, FastP and FastQ Screen version 0.12.0. The reads were aligned to the mouse genome and annotation (GRCm38.92 version) using HiSat2 version 2.1.0183. Determination and statistical analysis of expression levels was done by a combination of HTSeq version 0.9 .1184 , the R environment version 3.4, utilizing packages from Bioconductor and differential gene expression analysis based on the negative binomial distribution using the DESeq2 package version 1.18.1186. Pathway Analysis was preformed using MetaCore from Clarivate Analytics (https://portal.genego.com/).

\section{Single cell RNA sequencing on kidneys}

$3 \Delta \mathrm{Mdm} 2^{\mathrm{Hep}}$ and 3 control mice were culled by $\mathrm{CO}_{2}$ inhalation, blood samples were collected by cardiac puncture and $30-40 \mathrm{ml}$ cold PBS were used to perfuse the circulatory system via the left heart. The 6 mice were culled, and their kidneys processed, on 2 different days. 2 mice ( $1 \Delta \mathrm{Mdm} 2^{\mathrm{Hep}}$ and 1 control) were culled together on one day and the others $\left(2 \Delta \mathrm{Mdm} 2^{\mathrm{Hep}}\right.$ and 2 control) were culled 2 months later. On each occasion, the left kidney was used for dissociation and generation of single-cell suspension, while the right kidney was fixed in $10 \%$ NBF. The renal capsule of the left kidney was removed, the kidney was cut into equally-sized pieces and was dissociated using the Multi-tissue dissociation kit 1 (Miltenyi, 130110-201) as per the manufacturer's instructions. $0.25 \mathrm{~g}$ of kidney tissue were placed in a GentleMACS C tube with dissociation buffer ( $2.35 \mathrm{ml}$ serum-free RPMI culture medium (Gibco), $100 \mu$ l enzyme D, 50 $\mu \mathrm{l}$ enzyme R and $12.5 \mu$ l enzyme A). Kidney dissociation was performed in a GentleMACS dissociator using the "heart_01_01" programme (15s). The samples were placed in a waterbath $\left(37^{\circ} \mathrm{C}\right)$ for 30 minutes and then placed back in the dissociator ("heart_01_02" programme, 30s).

After the second round of dissociation, $8 \mathrm{ml}$ of sterile PBS/10\% FBS were added in the C tubes to stop the reaction. The samples were passed through a $40 \mu \mathrm{m}$ cell strainer into a $50 \mathrm{ml}$ falcon tube. All subsequent steps were performed on wet ice or at $4^{\circ} \mathrm{C}$. The samples were spun at $300 \mathrm{~g}$ for 5 mins and re-suspended in $5 \mathrm{ml}$ cold PBS. They were spun again at $300 \mathrm{~g}$ for $5 \mathrm{mins}$, re-suspended in $1 \mathrm{ml}$ red blood cell lysis buffer (8.29 $\mathrm{g} \mathrm{NH}_{4} \mathrm{Cl}, 1 \mathrm{~g} \mathrm{KHCO}_{3}$ and $37.2 \mathrm{~g} \mathrm{Na}_{2}$ EDTA in PBS) and incubated for 30 sec on wet ice. The samples were topped with $9 \mathrm{ml}$ of cold PBS and washed twice, as described previously (spin at $300 \mathrm{~g}$ for 5 mins and re-suspended in PBS). The samples were re-suspended in cold PBS, were subjected to debris removal using the debris removal solution (Miltenyi) according to the manufacturer's instructions. Finally, the samples were re-suspend in $10 \mathrm{ml}$ cold PBS/10\% FBS/2.5mM EDTA.

Cell viability and concentration were determined using the Trypan Blue assay and flow cytometry (viability $\geq 90 \%$ ). 20,000-40,000 cells were loaded on a 10x Chromium chip (1 sample per lane). Cleanup, reverse transcription, cDNA amplification and library preparation were performed using the Chromium Single cell 3' Reagent Kits (v3) as per the manufacturer's instructions.

The samples were sequenced in the Illumina NextSeq 500 using the $2 \times 150 \mathrm{bp}$ kit with the following read length parameters: 26bp Read1 - cell barcode and UMI, 8bp 17 index - sample index, 98bp Read2 - 
transcript read. CellRanger v.4.0 (with default parameters) was used to demultiplex Illumina BCL output files and align reads to the ensemble GRCm38.99 reference genome with the addition of the AAV8-TBGCre and AAV8-TBG-Null sequences.

All other steps were performed using R v.4.0 and packages from Bioconductor v.3.12. Raw matrices generated by CellRanger were transformed to SingleCellExperiment (SCE) objects and they were filtered to exclude empty droplets using the DropletUtils v.1.10 package. SCE objects were merged together to perform further downstream analysis. Following similar parameters used in a previous scRNA-seq analysis murine kidney ${ }^{33}$, poor quality cells ( $<75$ or $>3000$ expressed genes or $>50 \%$ mitochondrial gene expression) were filtered out and only genes expressed in more than 10 cells with at least one UMI were kept for further analysis.

The normalisation by deconvolution method designed by Lun et al. ${ }^{34}$ was used to normalise and log transform the counts with functions from Scater package v.1.18. Highly variable genes were computed with default parameters and the top $10 \%$ were used to perform principal component analysis (PCA) and Uniform Manifold Approximation and Projection (UMAP) using the top 20 PCs. After examining UMAP plots coloured by batch run, it was determined that batch correction was not required.

Initial clustering was performed with functions from the Seurat package v.4.0 with default parameters and with eps value of 0.5 and resolution sequence of 0.1 to 1 by 0.1 . Markers of each cluster were identified by performing a pairwise differential analysis between each pair of clusters with a minimum difference of $20 \%$ of cells expressing the gene and log 2 fold change threshold of $>0.25$ and only keeping the differentially expressed markers in all the comparisons. Re-clustering of control cells with a new PCA and UMAP was performed and the main markers were used to manually classify clusters into different cell types. $\triangle M d m 2^{\text {Hep }}$ cells were projected onto the reference UMAP and assigned to the identified clusters. The same methodology was followed for the identified control Proximal Tubular Cells (PTCs), Distal Tubular Cells (DTCs) and the initial cluster "Mesenchymal cells" for the projection and assignment of the $\Delta M d m 2^{\text {Hep }}$ cells. Scores for senescence, p21, proliferation, TGF $\beta$, JAK-STAT and regeneration signatures were computed using the AddModuleScore Seurat function. The genes included in each list used to create the signatures can be found on Supplemental Table 3. All transcriptomic data will be made publicly available on GEO (GSE189726) at the time of publication.

DGE analysis was performed as described by Giustacchini et al. $2017^{35}$. Briefly, $\log _{2}$ fold change was calculated between groups and non-parametric wilcoxon test was used to compare the expression values. Fisher's exact test was used to compare expressing cell frequency (percentage of cells per group with at least one UMI count). P-values from both tests were combined using Fisher's method and adjusted using Benjamini-Hochberg. Genes were considered to be differentially expressed if the $\mathrm{p}$ adjusted values were $<0.05$. Heatmaps comparing relative gene expression by cell groups were computed using the "DoHeatmap" function from Seurat R package or "plotGroupedHeatmap" function from the scater $\mathrm{R}$ package. In both cases, the scaled value from each group was calculated by 
substracting the average logcount gene expression from the total mean gene expression and divided by the standard deviation. A two colour range scale was used to convert scale values into colour intensity.

\section{Protein extraction and western blotting}

Snap frozen tissue was homogenized in $300 \mu$ of protein extraction buffer $(50 \mathrm{mM}$ HEPES, $100 \mathrm{mM} \mathrm{NaF}$, $150 \mathrm{mM} \mathrm{NaCl}, 10 \mathrm{mM} \mathrm{Na}_{4} \mathrm{P}_{2} \mathrm{O}_{7}, 10 \mathrm{mM}$ EDTA, $1 \%$ Triton X100, 0.1\% SDS, 0.5\% Na Deoxycholate in ddH2O), 1:100 Protease inhibitor (Thermo Fisher, 1862209) and 1:100 Phosphatase inhibitor (Thermo Fisher, 1862495) in $\mathrm{ddH}_{2} \mathrm{O}$ ) using the Precellys Evolution homogenizer (Bertin technologies). The lysate was passed through a $25 \mathrm{G}$ needle $5-8$ times and was then spun twice at $20800 \mathrm{~g}$ for 10 minutes at $4{ }^{\circ} \mathrm{C}$. The Bradford assay was used to measure protein concentration using the Coomasie Plus reagent (Thermo Fisher, 23236) on a 96-well plate setting. Absorbance was measured immediately at 596nm and a standard curve was automatically created using a Spectramax reader.

Western blotting was performed on precast gels using the XCell SureLoc Mini-Cell and XCell I Blot Module (Invitrogen, 10572913) protein samples were mixed with loading buffer (4X NuPAGE loading buffer (Invitrogen, 11549166) and 5\% $\beta$-mercaptoethanol) at a concentration of 20 $\mu \mathrm{g} / \mu \mathrm{l}$. The samples were heated for 5 minutes at $95^{\circ} \mathrm{C}$ and then were spun for 2 minutes at $20800 \mathrm{~g}$. $20 \mu \mathrm{l} \mathrm{sample} \mathrm{and} 5 \mu \mathrm{l}$ of protein ladder (Biorad, 1610373) were loaded on a 4 to 12\%, Bis-Tris, $1.0 \mathrm{~mm}$, 10-well precast gel (Invitrogen, NP0321PK2) and the gel was run at 120V for 1 hour and 50 minutes in MOPS running buffer (Invitrogen, NP-0001). Transfer onto PVDF membranes was performed by wet transfer, using the NuPAGE transfer buffer (Invitrogen, NP-0006) for 1 hour at 30V. Transfer efficiency was assessed by Ponceau S staining. The membranes were blocked for 1 hour with $5 \%$ BSA and then incubated with the primary antibody (diluted in $5 \% \mathrm{BSA}$ ) overnight at $4{ }^{\circ} \mathrm{C}$. This was followed by 45 minute incubation with the HRPconjugated secondary antibody and ECL incubation for the appropriate amount of time (2 minutes for pSmad2, pSmad3, Smad2 and Smad3 and 5 seconds for the b-actin). Visualisation of the bands was performed using the Chemidoc imaging system (Biorad).

\section{ELISA and cytokine arrays}

For the detection of Cystatin C in murine plasma, the "Mouse/Rat Cystatin C" Immunoassay kit (R\&D Technologies, MSCTC0) was used, according to the manufacturer's instructions. The plate was scanned in a plate reader at $450 \mathrm{~nm}$ (wavelength correction was set to $570 \mathrm{~nm}$ ) within 10 minutes after assay completion. Standard curve concentration calculations were performed on the "Myassays" website (https://www.myassays.com/).

Cytokine arrays on murine plasma and tissue samples were performed by Eve Technologies, using the TGF $\beta 1$, 2, 3 Magnetic bead kit for the measurement of the TGF $\beta$ ligands and the Milliplex MAP mouse cytokine magnetic bead panel (Discovery assay "Mouse Cytokine Array / Chemokine Array 31-Plex (MD31)"). 


\section{Metabolomics on mouse urine}

For the detection of urine amino acids, urine was collected from the mice by free urination 2 and 3 days before AAV-Cre injection, on injection day as well as 3 and 4 days post AAV-Cre injection. Urine collected by free urination after scruffing the mice was diluted 1:50 in cold metabolite extraction buffer $(50 \%$ methanol, $30 \%$ acetonitrile, $20 \%$ water) and were vortexed for 30 seconds. The samples were then centrifuged at $20800 \mathrm{~g}$ for 10 minutes at $4{ }^{\circ} \mathrm{C}$. LC-MS was performed as described previously ${ }^{36}$.

\section{Cell culture}

\section{Murine embryonic fibroblasts}

WT Murine embryonic fibroblasts (MEFs) were isolated from one E13-E14 WT C57BI/6 embryo. The MEFs were cultured on $10 \mathrm{~cm}$ petri dishes (Corning) in Dulbecco's modified eagle medium (DMEM) supplemented with $10 \%$ FBS, $1 \%$ penicillin/streptomycin, 1\% L-glutamine (CDMEM) at low 02 pressure $(3 \%)$. The MEFs cultures were confirmed to be free of mycoplasma contamination. For the plasma treatments, passage (P) 3-P5 MEFs were trypsinised and cell density was determined by using the CASY cell counter (Cambridge Bioscience, 5651808). 30000 cells (in $1 \mathrm{ml}$ medium) were plated on 24-well plate wells (with a round coverslip in each well) and were incubated with CDMEM with $1 \%$ plasma from either $\Delta \mathrm{Mdm} 2^{\mathrm{Hep}}$ or control mice for 24 hours. The cDMEM with plasma was changed with fresh cDMEM every 2 days and 6 days after the first media change the cells were stained for SA $\beta$-Gal as described above. The coverslips were mounted on slides and the SA $\beta-\mathrm{Gal}^{+}$cells were quantified by manual counting of 20 random fields of view of using a Zeiss Axiovert 200 microscope at 20x magnification.

\section{Induced Pluripotent Stem cell-derived neuronal cells}

Cells were derived from human fetal neural crest progenitors by Peter Hanson and Chris Morris at the University of Newcastle. The cells were passaged using trypsin-EDTA solution and were seeded into a geltrex coated 48 well cell culture plate at a density of 5,000 cells per well with $250 \mu$ l of proliferation media. They were allowed to proliferate for 2 days before the proliferation media was withdrawn and replaced with differentiation medium. Growth medium was replaced with differentiation medium for 16 days before beginning experimental procedure. The composition of proliferation and differentiation medium has been described previously ${ }^{37}$.

For the plasma treatment experiments, plasma samples were thawed on ice, vortexed and heat inactivated for 30 minutes at $60^{\circ} \mathrm{C}$. They were diluted in media to the desired concentration $(1: 100)$ and added to the plate for 24 hours. For the additional treatment with TGF $\beta R 1 i$, either TGF $\beta R 1 i(0.2 \mathrm{mM})$ or 
Vehicle (DMSO) were mixed with the plasma-containing medium and stayed on the cells for 24 hours. 2 days later the cells were stained for SA $\beta-G a l$ and the positive cells were quantified as described above.

\section{Statistical analyses and graphs}

The Prism 9 Software (GraphPad Software, Inc.) was used for statistical analyses. The Shapiro-Wilk test was used to assess data normality. For normally distributed data, the One-way ANOVA, 2-way ANOVA, the Brown-Forsythe and Welch ANOVA test, unpaired t-test or the Welch's t-test were used to test for statistical significance between data groups. The Kruskal-Wallis test or the Mann-Whitney test were performed for non-parametric data. All statistical tests comparing 2 groups were two-tailed. All figures were created using the Scribus Software (v1.4.7, G.N.U. general public licence). Unless otherwise stated, all data points on line graphs represent mean \pm Standard Error of Mean (S.E.M.). In bar graphs, bars represent mean \pm S.E.M and each dot represents a single mouse.

\section{References}

1. Coppé, J.-P., Desprez, P.-Y., Krtolica, A. \& Campisi, J. The Senescence-Associated Secretory Phenotype: The Dark Side of Tumor Suppression. Annu Rev Pathol 8, 99-118 (2010).

2. Muñoz-Espín, D. \& Serrano, M. Cellular senescence: from physiology to pathology. Nat. Rev. Mol. Cell Biol. 15, 482-496 (2014).

3. Baker, D. J. et al. Clearance of p16 Ink4a-positive senescent cells delays ageing-associated disorders. Nature 479, 232-236 (2011).

4. Baker, D. J. et al. Naturally occurring p16 Ink4a-positive cells shorten healthy lifespan. Nature 530, 184-189 (2016).

5. Xu, M. et al. Senolytics improve physical function and increase lifespan in old age. Nat. Med. 24, $1246-1256$ (2018).

6. Chang, J. et al. Clearance of senescent cells by ABT263 rejuvenates aged hematopoietic stem cells in mice. Nat. Med. 22, 78-83 (2016).

7. Bird, T. G. et al. TGF $\beta$ inhibition restores a regenerative response in acute liver injury by suppressing paracrine senescence. Sci. Transl. Med. 10, (2018).

8. Hoare, M. et al. NOTCH1 mediates a switch between two distinct secretomes during senescence. Nat. Cell Biol. 18, 979-992 (2016).

9. Acosta, J. C. et al. A complex secretory program orchestrated by the inflammasome controls paracrine senescence. Nat. Cell Biol. (2013) doi:10.1038/ncb2784. 
10. Teo, Y. V. et al. Notch Signaling Mediates Secondary Senescence. Cell Rep. 27, 997-1007.e5 (2019).

11. Ferreira-Gonzalez, S. et al. Paracrine cellular senescence exacerbates biliary injury and impairs regeneration. Nat. Commun. 9, 1-15 (2018).

12. Kiourtis, C. et al. Specificity and off-target effects of AAV8-TBG viral vectors for the manipulation of hepatocellular gene expression in mice. Biol. Open 1-11 (2021) doi:10.1242/bio.058678.

13. Bodoy, S. et al. Inducible slc7a7 knockout mouse model recapitulates lysinuric protein intolerance disease. International Journal of Molecular Sciences vol. 20 (2019).

14. Braun, D. et al. Aminoaciduria, but normal thyroid hormone levels and signalling, in mice lacking the amino acid and thyroid hormone transporter Slc7a8. Biochem. J. 439, 249-255 (2011).

15. Nagamori, S. et al. Novel cystine transporter in renal proximal tubule identified as a missing partner of cystinuria-related plasma membrane protein rBAT/SLC3A1. Proc. Natl. Acad. Sci. U. S. A. 113, 775-780 (2016).

16. Kang, H. M. et al. SOX9-Positive Progenitor Cells Play a Key Role in Renal Tubule Epithelial Regeneration in Mice. Cell Rep. 14, 861-871 (2016).

17. Kumar, S. et al. SOX9 Activation Highlights a Cellular Pathway of Renal Repair in the Acutely Injured Mammalian Kidney. Cell Rep. 12, 1325-1338 (2015).

18. Sturmlechner, I. et al. P21 Produces a Bioactive Secretome That Places Stressed Cells Under Immunosurveillance. Science 374, eabb3420 (2021).

19. Demaria, M. et al. An essential role for senescent cells in optimal wound healing through secretion of PDGF-AA. Dev. Cell 31, 722-733 (2014).

20. Hiebert, P. et al. Nrf2-Mediated Fibroblast Reprogramming Drives Cellular Senescence by Targeting the Matrisome. Dev. Cell 46, 145-161.e10 (2018).

21. Mosteiro, L., Pantoja, C., de Martino, A. \& Serrano, M. Senescence promotes in vivo reprogramming through p16INK4aand IL-6. Aging Cell 17, (2018).

22. Mosteiro, L. et al. Tissue damage and senescence provide critical signals for cellular reprogramming in vivo. Science (80-. ). 354, (2016).

23. Ritschka, B. et al. The senescence-associated secretory phenotype induces cellular plasticity and tissue regeneration. Genes Dev. 31, 172-183 (2017).

24. Ogrodnik, M. et al. Cellular senescence drives age-dependent hepatic steatosis. Nat. Commun. 8, 15691 (2017). 
25. Xu, M. et al. JAK inhibition alleviates the cellular senescence-associated secretory phenotype and frailty in old age. Proc. Natl. Acad. Sci. U. S. A. 112, E6301-E6310 (2015).

26. Ogrodnik, M. et al. Whole-body senescent cell clearance alleviates age-related brain inflammation and cognitive impairment in mice. Aging Cell 20, 1-16 (2021).

27. du Sert, N. P. et al. The arrive guidelines 2.0: Updated guidelines for reporting animal research. PLoS Biol. 18, 1-12 (2020).

28. Grier, J. D., Yan, W. \& Lozano, G. Conditional allele of mdm2 which encodes a p53 inhibitor. Genesis 32, 145-147 (2002).

29. Madisen, L. et al. A robust and high-throughput Cre reporting and characterization system for the whole mouse brain. Nat. Neurosci. 13, 133-140 (2010).

30. Jackson, E. L. et al. Analysis of lung tumor initiation and progression using conditional expression of oncogenic K-ras. Genes Dev. 15, 3243-3248 (2001).

31. Driver, J. E. et al. Impairment of hippocampal gamma (Y)-frequency oscillations in vitro in mice overexpressing human amyloid precursor protein (APP). Eur. J. Neurosci. 26, 1280-1288 (2007).

32. Dimri, G. P. et al. A biomarker that identifies senescent human cells in culture and in aging skin in vivo. Proc. Natl. Acad. Sci. U. S. A. 92, 9363-9367 (1995).

33. Park, J. et al. Single-cell transcriptomics of the mouse kidney reveals potential cellular targets of kidney disease. Science (80-. ). 360, 758-763 (2018).

34. Lun, A. T. L., Bach, K. \& Marioni, J. C. Pooling across cells to normalize single-cell RNA sequencing data with many zero counts. Genome Biol. 17, 1-14 (2016).

35. Giustacchini, A. et al. Single-cell transcriptomics uncovers distinct molecular signatures of stem cells in chronic myeloid leukemia. Nat. Med. 23, 692-702 (2017).

36. Voorde, J. Vande et al. Improving the metabolic fidelity of cancer models with a physiological cell culture medium. Sci. Adv. 5, (2019).

37. Madgwick, A. et al. Neural differentiation modulates the vertebrate brain specific splicing program. PLoS One 10, 1-14 (2015).

\section{Figures}




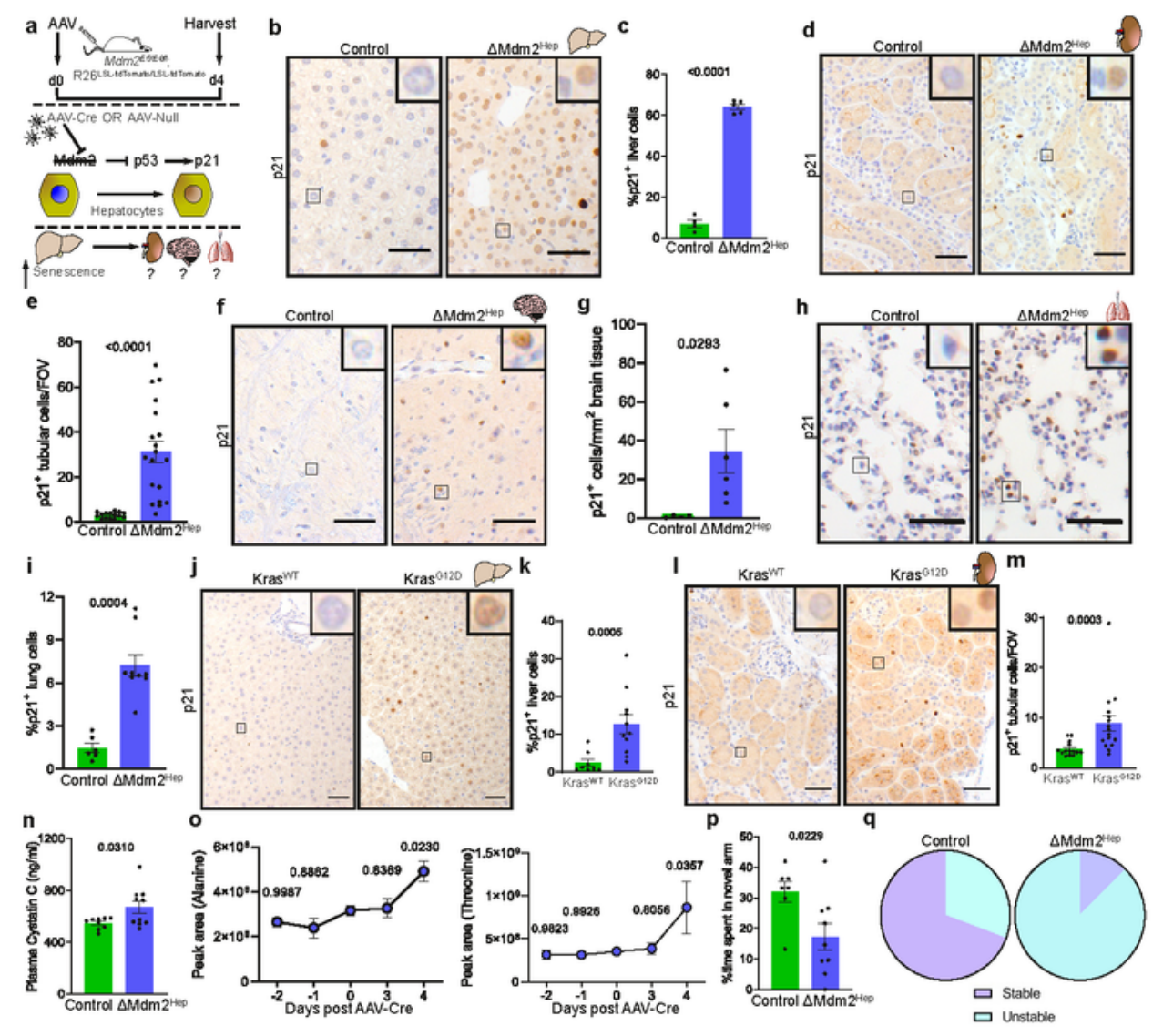

\section{Figure 1}

Hepatocellular senescence results in senescence and dysfunction in other organs. (a) Schematic of the

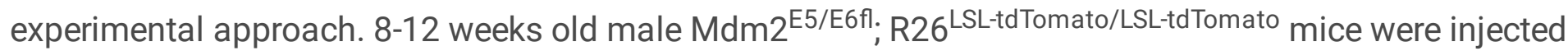
with either AAV8-TBG-Cre (AAV-Cre) or with AAV8-TBG-Null (AAV-Null) and culled 4 days later. Downstream targets of MDM2 in hepatocytes are highlighted. (b) Representative photos of p21 $\mathrm{IHC}$ on liver sections of $\Delta \mathrm{Mdm} 2^{\mathrm{Hep}}$ and control mice. (c) Automated quantification of $\mathrm{p} 21^{+}$liver cells; data are 
presented as percentage of total liver cells. $n=4$ and 5 control and $\Delta M d m 2^{\text {Hep }}$ mice respectively; unpaired t-test. (d) Representative photos of p21 IHC on kidney sections of $\Delta \mathrm{Mdm} 2^{\mathrm{Hep}}$ and control mice. (e) Manual quantification of $\mathrm{p} 21^{+}$renal tubular cells: data are presented as $\mathrm{p} 21^{+}$tubular cells per field of view (FOV), $\mathrm{n}=16$ and 19 control and $\Delta \mathrm{Mdm} 2^{\text {Hep }}$ mice respectively; Welch's t-test. (f) Representative photos of p21 IHC on brain sections of $\Delta \mathrm{Mdm} 2^{\mathrm{Hep}}$ and control mice. (g) Manual quantification of $\mathrm{p} 21^{+}$ brain cells: data are presented as $\mathrm{p} 21^{+}$cells per square millimetre $\left(\mathrm{mm}^{2}\right), \mathrm{n}=4$ and 6 control $\Delta \mathrm{Mdm} 2^{\mathrm{Hep}}$ mice respectively; Welch's t-test. (h) Representative photos of p21 $\mathrm{IHC}$ on lung sections of $\Delta \mathrm{Mdm} 2^{\mathrm{Hep}}$ and control mice. (i) Automated quantification of $\mathrm{p} 21^{+}$lung cells: data are presented as percentage of total lung cells, $\mathrm{n}=6$ control mice and $9 \Delta \mathrm{Mdm} 2^{\mathrm{Hep}}$ mice respectively; Unpaired t-test. (j) Representative photos of p21 IHC on liver sections of Kras ${ }^{\mathrm{G} 12 \mathrm{D}}$ and Kras ${ }^{\mathrm{WT}}$ mice. (k) Automated quantification of $\mathrm{p}^{2} 1^{+}$liver cells: data are presented as percentage of total liver cells, $\mathrm{n}=8$ and $11 \mathrm{Kras}^{\mathrm{WT}}$ and $\mathrm{Kras}^{\mathrm{G} 12 \mathrm{D}}$ mice respectively; Mann-Whitney test. (I) Representative photos of p21 IHC on kidney sections of Kras ${ }^{\mathrm{G} 12 \mathrm{D}}$ and Kras ${ }^{\mathrm{WT}}$ mice. (m) Manual quantification of $\mathrm{p} 21^{+}$renal tubular cells: data are presented as $\mathrm{p} 21^{+}$cells per FOV, $\mathrm{n}=14$ and $16 \mathrm{Kras}^{\mathrm{WT}}$ and $\mathrm{Kras}^{\mathrm{G12D}}$ mice respectively; Mann-Whitney test. (n) Plasma levels of Cystatin $\mathrm{C}$ in $\triangle \mathrm{Mdm} 2^{\mathrm{Hep}}$ and control mice 4 days post $\mathrm{AAV}$ injection, determined by ELISA. $\mathrm{n}=9$ and 10 control and $\triangle \mathrm{Mdm} 2^{\mathrm{Hep}}$ mice respectively; Welch's t-test. (o) Urine levels of Alanine and Threonine identified by liquid chromatography-mass spectrometry (LC-MS) in $\triangle \mathrm{Mdm} 2^{\mathrm{Hep}}$ mice pre- and post- AAV-Cre injection: dots represent the average peak area of $n=3$ mice at days -2 and $0, n=4$ mice at day 4 and $n=5$ mice at days -1 and 3; 2-way ANOVA comparing each time point to induction day (day 0 ). (p) Proportion of time spent by the $\Delta \mathrm{Mdm} 2^{\mathrm{Hep}}$ and control mice in the new arm of the $\mathrm{Y}$-maze at day 4 post AAV injection. $\mathrm{n}=7$ and 9 control and $\Delta \mathrm{Mdm} 2^{\mathrm{Hep}}$ mice respectively; unpaired t-test. (q) Pie charts showing the share of stable VS unstable oscillations of the hippocampal brain slices. $n=13$ brain slices from 4 control mice and $n=14$ slices from $4 \Delta \mathrm{Mdm} 2^{\mathrm{Hep}}$ mice. On all bar charts, bars are mean \pm S.E.M., each dot represents one sample from one mouse and the numbers are $p$ values. Scale bars are $50 \mu \mathrm{m}$. 


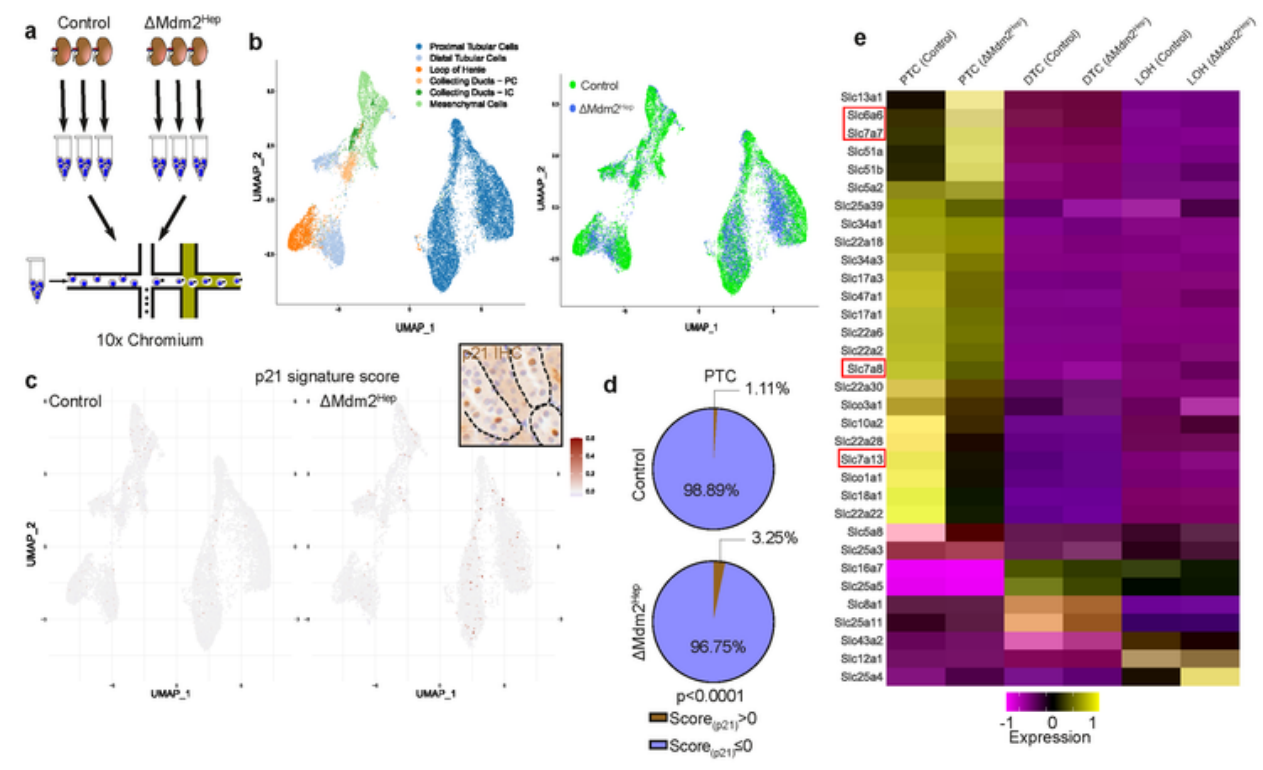

\section{Figure 2}

Single-cell RNA sequencing reveals significant transcriptional changes, including amino acid transporters within the proximal tubular compartment in response to liver senescence. (a) Schematic of the scRNA-seq experiment. 3 kidneys from control mice and 3 kidneys from $\Delta \mathrm{Mdm} 2^{\mathrm{Hep}}$ mice were dissociated and droplet-based scRNA-seq was performed on them on a 10x Chromium chip. (b) UMAP plots of all 24,215 cells (control and $\Delta \mathrm{Mdm} 2^{\mathrm{Hep}}$ ). Cells are coloured based on the broad clusters (left plot) or experimental 
group (right plot); green=control, blue $=\Delta M d m 2^{\text {Hep }}$. (c) UMAP showing the distribution of the cells that have a positive score for the p21 gene signature in the control (left) and the $\Delta \mathrm{Mdm} 2^{\mathrm{Hep}}$ (right) cells. Inset image is a representative kidney section stained for $\mathrm{p} 21$ by $\mathrm{IHC}$ with tubules highlighted (dashed lines). (d) Pie charts showing the share of $\mathrm{p} 21^{\text {high }}$ PTCs in the control and $\Delta M d m 2^{\text {Hep }}$ samples. Contingency was tested with the chi-square test. (e) Heatmap showing the significantly differentially expressed Slc transporter genes in the proximal tubular cell (PTC), distal tubular cell (DTC) and Loop of Henle (LOH) compartments. Gene IDs in red frames are transporters associated with amino acid transport. 

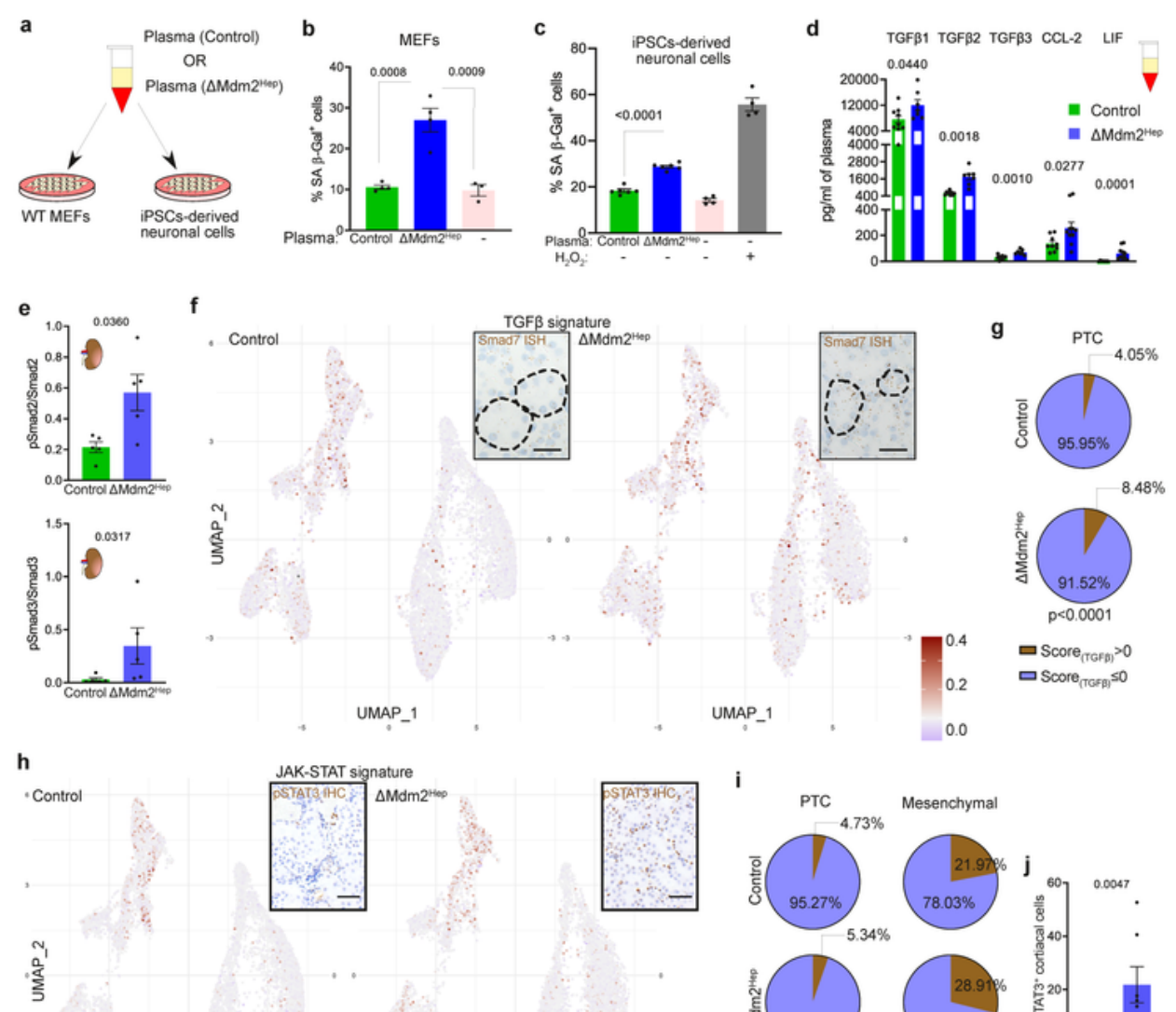

UMAP_1
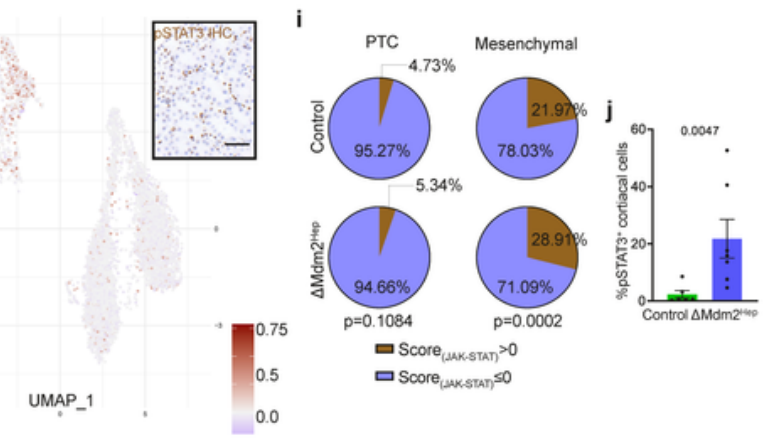

\section{Figure 3}

Increased concentration of circulating SASP factors is associated with senescence induction and increased renal TGF $\beta$ and JAK-STAT signalling. (a) Schematic of the in vitro plasma treatment. WT MEFs and iPSC-derived neuronal cells were treated with plasma from either control or $\triangle \mathrm{Mdm} 2^{\mathrm{Hep}}$ mice and were later stained for SA $\beta$-Gal to examine senescence induction. (b) Manual quantification of SA $\beta-\mathrm{Gal}^{+}$ WT MEFs; data are presented as percentage of total cells. Each dot represents one technical replicate 
(one 96 well plate well). $n=2$ technical replicates from each of the 2 biological replicates (plasma samples) from both control and $\triangle \mathrm{Mdm} 2^{\mathrm{Hep}}$ groups. $\mathrm{n}=3$ technical replicates for the "no plasma" group; one-way ANOVA. (c) Manual quantification of SA $\beta$-Gal' iPSC-derived neuronal cells, shown as percentage of total cells. Cells treated with $\mathrm{H}_{2} \mathrm{O}_{2}$ served as positive control for the stain. Each dot represents the average of a technical triplicate ( 3 wells) of one biological replicate (plasma sample) for both plasma-treated groups. For the "no plasma" groups (with and without $\mathrm{H}_{2} \mathrm{O}_{2}$ ), each dot is a technical replicate. $n=6$ for both the control and $\Delta M d m 2^{\text {Hep }}$ groups and $n=4$ for the "no plasma" groups. One-way ANOVA. (d) Plasma levels of transforming growth factor $\beta$ (TGF) $\beta 1$, TGF $\beta 2$, TGF $\beta 3$, C-C motif chemokine ligand 2 (CCL2) and leukaemia inhibitory factor (LIF), measured by cytokine arrays. For the TGF $\beta$ ligands, $\mathrm{n}=9$ control plasma samples and $\mathrm{n}=7 \Delta \mathrm{Mdm} 2^{\mathrm{Hep}}$ plasma samples. For CCL2, $\mathrm{n}=9$ control plasma samples and $n=10 \Delta M d m 2^{\text {Hep }}$ plasma samples. For $L I F, n=7$ control plasma samples and $n=10$ $\Delta M d m 2^{\text {Hep }}$ plasma samples. Unpaired t-test (TGF 1 and 3), Welch's t-test (TGF $\beta 2$ and CCL2) and MannWhitney test (LIF). Each dot represents one biological replicate (one plasma sample from one mouse). (e) Quantification of signal intensity of the western blot bands shown in Fig. S8b. $n=5$ for each group; Welch's t-test (pSMAD2/SMAD2) or Mann-Whitney test (pSMAD3/SMAD3). (f) UMAP showing the distribution of the cells that have a positive score for the TGF $\beta$ gene signature in the control (left) and the $\triangle \mathrm{Mdm} 2^{\mathrm{Hep}}$ (right) cells. Inset images are representative kidney sections stained for Smad7 by ISH (RNAscope), dash lines highlight renal tubules. (g) Pie charts showing the share of PTCs with a positive TGF $\beta$ signature score in the control and $\Delta M d m 2^{\text {Hep }}$ samples; contingency was tested with the chi-square test. (h) UMAP showing the distribution of the cells that have a positive score for the JAK-STAT gene signature in the control (left) and the $\triangle \mathrm{Mdm} 2^{\mathrm{Hep}}$ (right) cells. Inset images are representative kidney sections stained for pSTAT3 by IHC. (i) Pie charts showing the share of PTCs and mesenchymal cells with a positive JAK-STAT signature score in the control and $\triangle \mathrm{Mdm} 2^{\mathrm{Hep}}$ samples; contingency was tested with the chi-square test. (j) Automated quantification of pSTAT3 $^{+}$renal cortical cells; data are presented as percentage of total cortical cells, $n=6$ and 7 control and $\Delta M d m 2^{\text {Hep }}$ mice respectively; Mann-Whitney test. Bars are mean \pm S.E.M. and the numbers on the graphs are $p$ values. Scale bars are $50 \mu \mathrm{m}$. 

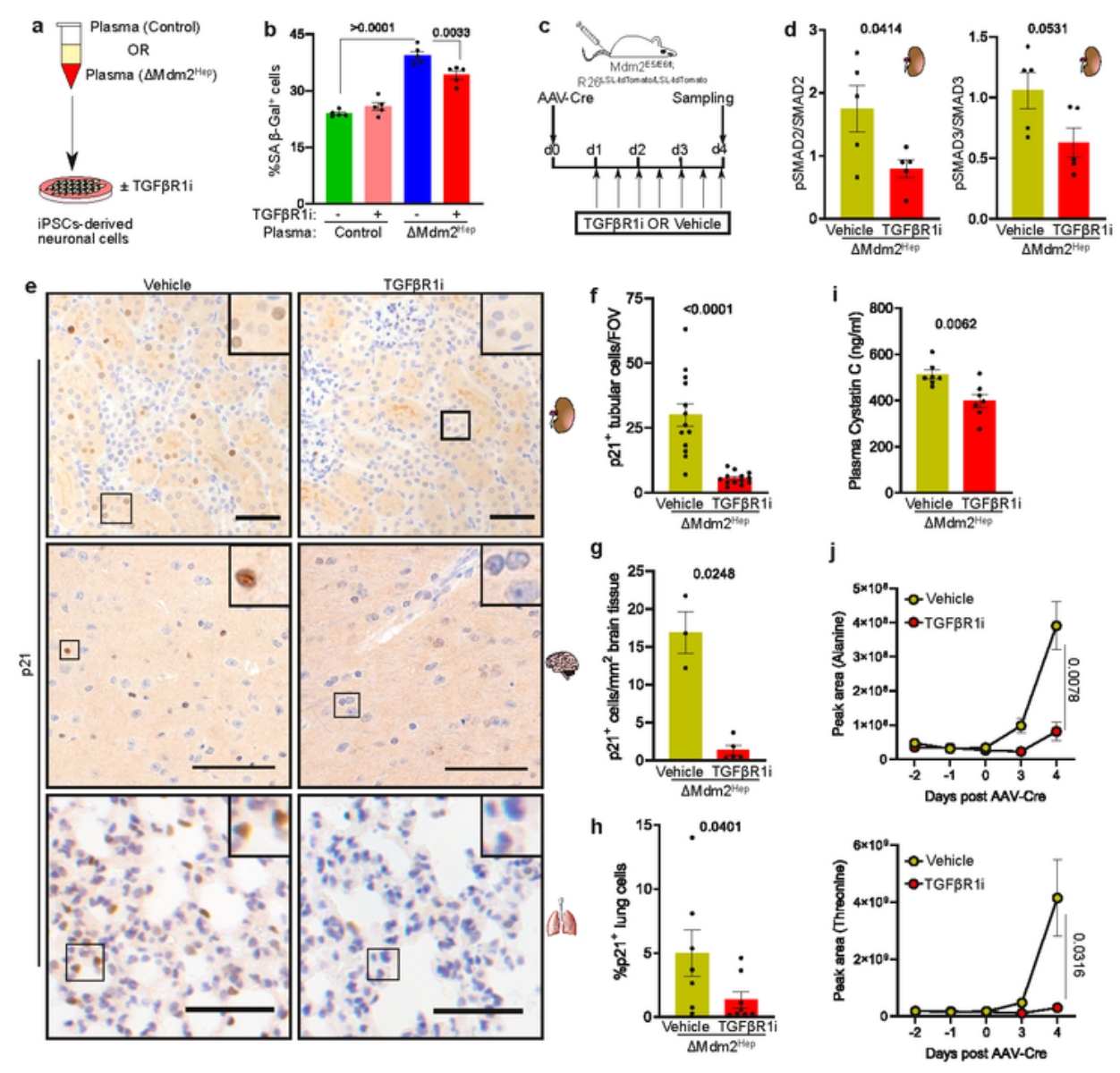

\section{Figure 4}

Inhibition of TGF $\beta$ signalling prevents induction of senescence in the extra-hepatic organs. (a) Schematic of the in vitro treatment with TGF $\beta$ inhibitor. Plasma-treated iPSC-derived neuronal cells were treated with either TGFßR1i (AZ12601011, AstraZeneca) or Vehicle (DMSO) and were later stained for SA $\beta$-Gal to assess senescence. (b) Manual quantification of SA $\beta-\mathrm{Gal}^{+}$cells, shown as percentage of total cells. Each dot represents the average of a technical triplicate from one biological replicate (plasma sample from one 
mouse), $n=5$ plasma samples for both control and $\triangle M d m 2^{\text {Hep }}$ (the same plasma sample was used for the TGFBR1i treatment in separate wells); one-way ANOVA. (c) Schematic of the in vivo treatment with TGF $\beta$ inhibitor. $\triangle M d m 2^{\text {Hep }}$ mice were treated with TGF $\beta R 1 \mathrm{i}$ or Vehicle $(0.5 \% \mathrm{HPMC})$ by oral gavage twice daily, starting 24 hours post AAV-Cre injection. (d) Quantification of signal intensity of the western blot bands shown in Fig. S9c. n=5 for each group; unpaired t-test for both datasets. (e) Representative photos of p21 IHC on kidney, brain and lung sections of $\Delta \mathrm{Mdm} 2^{\mathrm{Hep}}$ mice treated with either vehicle or with TGFBR1i. (f) Manual quantification of $\mathrm{p} 21^{+}$renal cortical cells: data are presented as $\mathrm{p} 21^{+}$cells per FOV. Each dot represents one mouse, $\mathrm{n}=13$ mice for each group; Mann-Whitney test. (g) Manual quantification of $\mathrm{p} 21^{+}$brain cells: data are presented as $\mathrm{p} 21^{+}$cells per square millimetre $\left(\mathrm{mm}^{2}\right), \mathrm{n}=3$ and 5 vehicle- and TGFBR1i-treated $\triangle \mathrm{Mdm} 2^{\mathrm{Hep}}$ mice respectively; Welch's t-test. (h) Automated quantification of $\mathrm{p} 21^{+}$lung cells; data are presented as percentage of total lung cells, $n=7$ and 8 vehicle- TGF $\beta R 1$ i-treated $\Delta M d m 2^{\text {Hep }}$ mice respectively; Mann-Whitney test. (i) Plasma Cystatin C of Vehicle- and TGF $\beta R 1$ i-treated $\triangle M d m 2^{H e p}$ mice at cull. Each dot represents one mouse, $n=7$ mice for each group; unpaired t-test. (j) Levels of Alanine and Threonine in the urine of $\Delta \mathrm{Mdm} 2^{\mathrm{Hep}}$ mice before and after $\mathrm{AAV}$-Cre injection, $\mathrm{n}=4$ and 5 Vehicle-and TGFBR1i-treated $\triangle M d m 2^{\text {Hep }}$ mice, respectively, per time point; Welch's t-test comparing Vehicle- to TGFBR1i-treated mice at day 4. Bars are mean \pm S.E.M. and the numbers on the graphs are $p$ values. Scale bars are $50 \mu \mathrm{m}$.

\section{Supplementary Files}

This is a list of supplementary files associated with this preprint. Click to download.

- Sup.Table1.xlsx

- Sup.Table2.xlsx

- Sup.Table3.xIsx

- Supplementalfigurelegends.docx

- Fig.S1.pdf

- Fig.S2.pdf

- Fig.S3.pdf

- Fig.S4.pdf

- Fig.S5.pdf

- Fig.S6.pdf

- Fig.S7.pdf

- Fig.S8.pdf

- Fig.S9.pdf

- Fig.S10.pdf 